\title{
Modification of DC Flashover Voltage at High Altitude on the Basis of Molecular Gas Dynamics
}

\author{
Dong-Ming Liu*, Fu-Sheng Guo ${ }^{\dagger}$ and Wen-xia Sima**
}

\begin{abstract}
The effect of altitude on thermal conduction, surface temperature, and thermal radiation of partial arc was investigated on the basis of molecular gas dynamics to facilitate a deep understanding of the pollution surface discharge mechanism. The DC flashover model was consequently modified at high altitude. The validity of the modified DC flashover model proposed in this paper was proven through a comparison with the results of high-altitude simulation experiments and earlier models. Moreover, the modified model was found to be better than the earlier modified models in terms of forecasting the flashover voltage. Findings indicated that both the thermal conduction coefficient and the surface thermodynamics temperature of partial arc had a linear decrease tendency with the altitude increasing from $0 \mathrm{~m}$ to $3000 \mathrm{~m}$, both of which dropped by approximately $30 \%$ and $3.6 \%$, respectively. Meanwhile, the heat conduction and the heat radiation of partial arc both had a similar linear decrease of approximately $15 \%$. The maximum error of DC pollution flashover voltage between the calculation value according to the modified model and the experimental value was within $6.6 \%$, and the pollution flashover voltage exhibited a parabola downtrend with increasing of pollution.
\end{abstract}

Keywords: Flashover, High altitude, Molecular gas dynamics, Energy balance model, Radiation

\section{Introduction}

The distribution of primary energy is reasonably and fully utilized in China because the abundant natural resources in the western region are transported to the eastern region through the UHV transmission lines that are being gradually put into operation. However, the terrain of China in the west is higher than that in the east, and regions in which the altitude exceeds $1000 \mathrm{~m}$ account for over $2 / 3$ of the whole land [1]. Owing to the rarefied air, cold climate, and different pollution degrees in high-altitude areas, electric insulation performance lower than that in plain areas. This condition threatens the safe operation of electric power systems.

To conduct an in-depth study on the physical mechanism of the pollution surface discharge and to identify basic measures to solve pollution flashover, M El-A Slama [2] conducted a systematic study on earlier pollution flashover models $[3,4]$ and found that the characteristic constant depended on the equivalent circuit parameters and the heat characteristics of the discharge channel. The effect of the insulated surface on gas discharge was investigated by Fusheng Guo [5], and the development model of the

$\dagger$ Corresponding Author: Yantai Electric Power Bureau, China. (guofs2012@sina.com)

* Yantai Electric Power Bureau, China. (13853589929@139.com)

** State Key Laboratory of Power Transmission Equipment \& System Security and New Technology, Chongqing University, China. (cqsmwx@cqu.edu.cn)

Received: May 24, 2014; Accepted: October 13, 2014 streamer at arc head was built. Wenxia Sima [6] proposed a DC radial development model along the pollution surface with consideration of the effect of energy radiation on flashover during arc development. In addition, the force on the arc development along the surface was analyzed, and a force analysis-based calculation model of arc velocity was proposed [7]. However, these studies did not consider the effect of atmospheric parameters on insulator surface discharge characteristics under contamination conditions, and research on how these models can be correctly remains insufficient $[8,9]$. In particular, a significant difference is observed when the correction coefficient of pollution flashover voltage is determined at high altitudes [10-13].

In this study, the effect of altitude on the thermal conduction, surface temperature, and thermal radiation of a partial arc was investigated on the basis of the arc energy balance model with consideration of radiation and gas molecular dynamics. The modified DC flashover model at high altitude was proposed and compared with other modified models in terms of the expression of thermal conduction and radiation of partial arc. The process of pollution surface discharge at high altitude was comprehensively investigated from a microcosmic perspective, which facilitated a deeper understanding of the physics parameters of surface discharge. This finding also has academic significance and engineering application value for discovering the discharge mechanism under complex conditions and for preventing flashover accidents. 


\section{Arc Energy Balance Model Considering Radiation}

Once the dry band is formed, most of the applied voltage is applied to it. The discharge at the location in which the electric field is concentrated between the electrode and insulation boundary is enhanced, and several steamers form a large discharge domain. When the charged particle grows to a specific concentration range, the volume force effect of plasma is enhanced, and the charged particles concentrate in the cylinder channel [14]. The highconcentration charged particles can enhance collision and composition in the channel, where a large amount of heat and light are sent, and a bright partial arc is formed. With the development of discharge, the arc is gradually extended forward across the dry band, and flashover is formed at the end. In this study, the Obenaus flat model was adopted, as shown in Fig. 1.

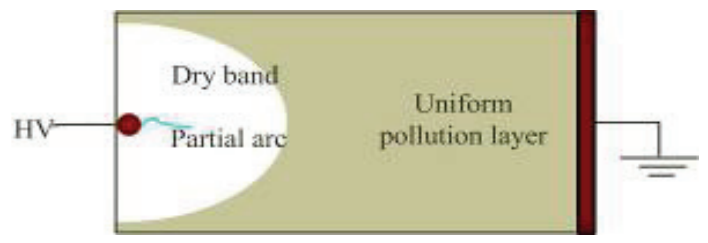

Fig. 1. Flat plate model of the polluted insulator

High-temperature radiation is an important mode of heat transmission. Arc temperature can reach $5000 \mathrm{~K}$ to 14000 $\mathrm{K}$ from its production to flashover [15]. Thus, the effect of the arc heat radiation is especially important to maintain heat balance. A high-temperature object will radiate energy outward by sending an electromagnetic wave without any medium while the radiation and absorption of the gas is occurring internally, which is irrelevant to the shape [16].

In this work, the partial arc is equal to a resistance that can send out radiation. With the enhanced discharge, the arc heat radiation is gradually enhanced. Based on the conservation of energy law and ignoring the effect of convection, total power is equal to the arc radiation, arc heat transmission, and the surplus pollution layer heat loss powers, which satisfies the energy conservation Eq. (1):

$$
U I=F_{\lambda} \varepsilon \delta T_{s}^{4} \cdot 2 \pi r x+K \frac{d T_{s}}{d r} \cdot 2 \pi r x+r_{c}(L-x) I^{2}
$$

where $T_{s}$ is the arc surface temperature; $r_{c}$ is the equivalent resistivity of the residual pollution layer, streamer, and air; $x$ is the arc length; $L$ is the insulating plate length; $\varepsilon$ is the grey body factor, $\varepsilon \in(0,1) ; F_{\lambda}$ is the blackbody radiation, which is function of the product of wavelength and arc surface temperature [17]; $\delta$ is the Stefan-Boltzmann Constant, $\delta=5.6705 \times 10^{-8} \mathrm{~W} \cdot \mathrm{m}^{-2} \cdot \mathrm{K}^{-4}$; and $\mathrm{K}$ is the $\operatorname{arc}$ thermal-conductivity coefficient.

Suppose that the heat conduction loss is caused by the heat production of arc plasma resistance, Eq. (1) can be expressed as (2):

$$
U I=F_{\lambda} \varepsilon \delta T_{s}^{4} \cdot 2 \pi r x+\frac{I^{2}}{\sigma\left(T_{c}\right)} x+r_{c}(L-x) I^{2}
$$

where $T_{c}$ is the arc center temperature, and $\sigma\left(T_{c}\right)$ is the arc conductivity.

During the development process, the arc current and radius meet the empirical Eq. [18] (3):

$$
r=\sqrt{\frac{I}{1.45 \pi}}
$$

Substituting Eq. (3) into the first term on the right in Eq. (2). The term of radiation energy can be expressed as (4):

$$
F_{\lambda} \varepsilon \delta T_{s}^{4} \cdot 2 \pi r x=C \sqrt{I} x
$$

where $\mathrm{c}=(4 \pi / 14500)^{1 / 2} F_{\lambda} \delta T_{S}^{4}$, which is defined as the arc radiation constant to characterize the radiation capacity sent forth to the surrounding and is the function of electromagnetic radiation wavelength, gray body factor, and arc surface temperature.

Substituting the Eq. (4) into Eq. (2), the critical flashover voltage can be obtained:

$$
U_{c}=C^{2 / 3} L r_{c}^{1 / 3}
$$

\section{Effect of Altitude on the Heat Exchange of Partial Arc}

\subsection{Effect of altitude on the thermal conduction of partial arc}

The contact area of two gases $A$ and $B$, which are in contact with each other at different temperatures $\left(T_{A}<T_{B}\right)$, is $d S$, as shown in Fig. 2. The average thermal motion energy of gas molecules of $A$ is lower than that of $B$ from a molecular dynamics perspective. The exchange between parts $A$ and $B$ is caused by thermal motion. Some gas molecules with high kinetic energy are transported from

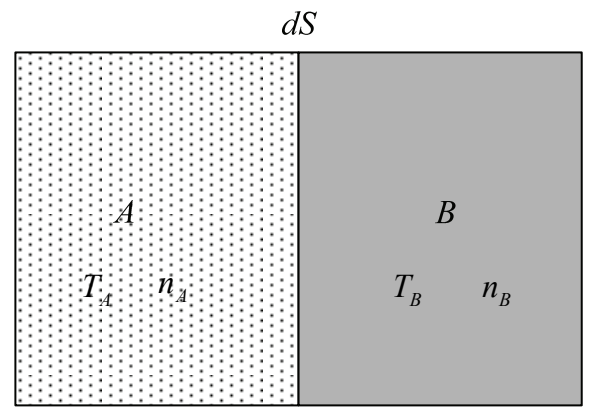

Fig. 2. Sketch map of heat exchange in gas 
$B$ to $A$, whereas others with low kinetic energy are transported from $A$ to $\mathrm{B}$, such that heat conduction is formed macroscopically.

Suppose that the gas molecule density and the average velocity of $A$ and $B$ are $n_{A}, n_{B}, v_{A}$, and $v_{B}$, respectively, which can be similarly regarded as $n_{A} v_{A}=n_{B} v_{B}=n v$. The irregular movement of gas molecules is simplified to move parallel to the $x, y$, and $z$-axes, and the number of gas molecules transported between $A$ and $B$ at $d t$ in arbitrary volume is obtained by:

$$
d N=\frac{1}{6} n v d S d t
$$

The energy transferred between $A$ and $B$ at $d t$ can be expressed as:

$$
\Delta Q=\frac{a+b+2 c}{2} k\left(T_{A}-T_{B}\right)
$$

where $a, b$, and $c$ are the translation freedom, rotation freedom, and vibration freedom of gas molecules, respectively; and $k$ is the Boltzmann constant.

The constant-volume specific heat of the gas is expressed as follows:

$$
c_{v}=\frac{(a+b+2 c) N k}{2 M}
$$

where $M$ is the gas quality, and $N$ is the number of molecules.

Substituting Eq. (8) into Eq. (7), we can obtain the energy transferred between $A$ and $B$ for one gas molecule at $d t$ as follows:

$$
\Delta Q=c_{v} m\left(T_{A}-T_{B}\right)
$$

Based on Eqs. (6) and (9), the total energy transferred between $A$ and $B$ at $d t$ can be obtained by:

$$
d Q=\frac{1}{6} \rho v c_{v}\left(T_{A}-T_{B}\right) d S d t
$$

where $\rho=n m$ is the gas molecule density, and $m$ is the gas molecule quality.

Hence, the heat energy transported is proportional to the temperature difference, contact area of the two parts of gases, and the transport time [19]. The average velocity of gas molecules with temperature $T$ is:

$$
v=\sqrt{\frac{8 k T}{\pi m}}
$$

Substituting Eq. (11) into Eq. (10) and compare it with the macroscopic thermal conductivity coefficient, we obtain the thermal conductivity coefficient at low air pressure, which is expressed as follows:

$$
\frac{n}{n_{0}}=\frac{p}{p_{0}} \times \frac{273+t_{0}}{273+t}
$$

The atmospheric pressure, temperature, and relative air density changed consistently with increments of altitude [20], as shown in Table 1 . The normal condition is $t=20 \mathrm{C}$, $p_{0}=101.3 \mathrm{kPa}$, and the modified method can be referred to GB311.2 83 or IEC60-1 73 under non-standard conditions. Thus, the relationship among relative air density, pressure, and temperature is expressed as follows:

$$
\frac{n}{n_{0}}=\frac{p}{p_{0}} \times \frac{273+t_{0}}{273+t}
$$

Given that no relationship exists between the temperature and the constant-volume specific heat of ideal gas, the ratio of thermal conduction coefficient at low pressure and atmospheric pressure can be obtained as:

$$
\frac{K}{K_{0}}=\frac{p}{p_{0}} \cdot \sqrt{\frac{T_{0}}{T}}
$$

According to Eq. (14) and Table 1, the variations of air thermal conduction coefficient with respect to the altitude is obtained as shown in Fig. 3. The thermal conduction coefficient of air obviously exhibited a linear decrease with increments in altitude, and a higher altitude resulted in a larger drop in the heat conduction coefficient. The thermal conduction coefficient dropped by $30 \%$ when the altitude increased from $0 \mathrm{~m}$ to $3000 \mathrm{~m}$.

Table 1. Variations of air pressure, relative air density, and temperature with the increments of altitude

\begin{tabular}{c|c|c|c|c}
\hline Altitude $(H / \mathrm{m})$ & 0 & 1000 & 2000 & 3000 \\
\hline Air pressure $(p / \mathrm{kPa})$ & 101.3 & 89.7 & 79.4 & 70.4 \\
\hline Relative air density & 1 & 0.901 & 0.812 & 0.732 \\
\hline Temperature $\left(T /{ }^{\circ} \mathrm{C}\right)$ & 20 & 14 & 8 & 2 \\
\hline
\end{tabular}

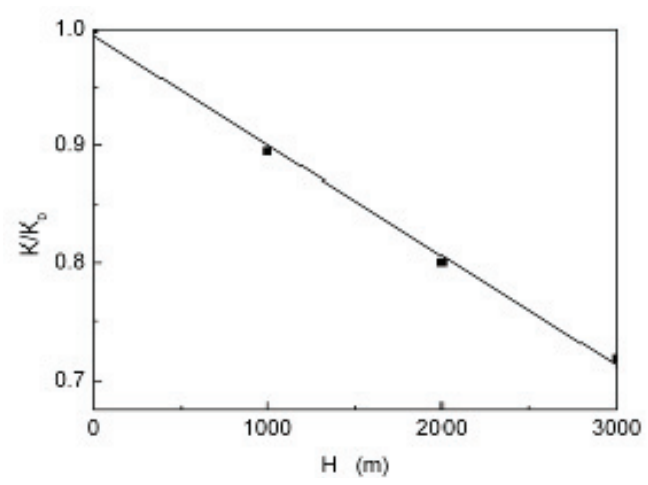

Fig. 3. Relationship between thermal conductivity coefficient and altitude 


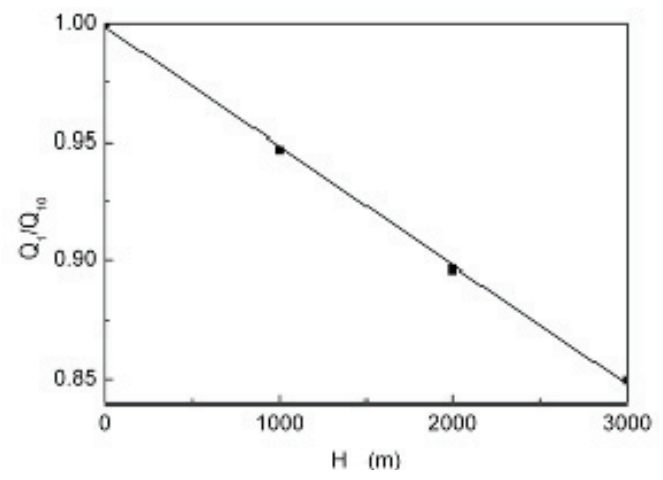

Fig. 4. Relationship between arc thermal conductivity value and altitude

Supposing that the temperature gradient $d T / d r$ of the arc edge is constant, and considering the thermal conduction formula and the variations of the arc radius with the air pressure [8], the ratio of the arc thermal conduction under low air pressure and under atmospheric pressure at the same current can be expressed as follows:

$$
\frac{Q_{1}}{Q_{10}}=\left(\frac{p}{p_{0}}\right)^{0.535} \sqrt{\frac{T_{0}}{T}}
$$

The thermal conduction capacity of the arc exhibited a linear decrease with increments in altitude, which agrees with the variations of arc thermal conduction with altitude, as shown in Fig. 4. The thermal conduction value of the arc dropped to approximately $85 \%$ of the atmospheric pressure when the altitude rose to $3000 \mathrm{~m}$. This drop indicates that the thermal conduction capacity of the arc was reduced at low pressure, and a small amount of energy from the power supply can maintain the forward movement of the arc.

\subsection{Effect of altitude on the partial arc surface tem- perature}

The currents through the arcs under a standard atmosphere and low air pressure are $I_{0}$ and $I$, respectively. For the same pollution and applied voltage, the arc radius and current meet the relationship as follows $[8,21]$ :

$$
\begin{gathered}
r_{0}=\sqrt{\frac{I_{0}}{1.45 \pi}} \\
r=\left(\frac{p}{p_{0}}\right)^{-0.465} \sqrt{\frac{I}{1.45 \pi}}
\end{gathered}
$$

According to Eqs. (16) and (17), the ratio of the current density at this two kind conditions can be expressed as follows:

$$
\frac{j}{j_{0}}=\left(\frac{p}{p_{0}}\right)^{0.93}
$$

By combining Eq. (18) with the micro expression of the current density in Eq. (19) and supposing that the electrons in the arc having the same velocity, the ratio of the arc current density at low air pressure and atmospheric pressure can be expressed as Eq. (20):

$$
\begin{aligned}
& j=n_{e} e v_{e} \\
& \frac{j}{j_{0}}=\frac{n_{e}}{n_{e 0}}
\end{aligned}
$$

Therefore, by comparing Eqs. (18) and (20), the ratio of the arc electron density in the two cases can be expressed as follows:

$$
\frac{n_{e}}{n_{e 0}}=\left(\frac{p}{p_{0}}\right)^{0.93}
$$

Given that the collision frequency in the plasma is proportional to the neutral background gas density and that the main collision is electron to neutral particle, the collision frequency between the electron and gas neutral particles can be considered proportional to the neutral gas molecules at room temperature:

$$
\gamma_{e m}=6.3 \times 10^{-9} n
$$

where $\gamma_{e m}$ is the collision frequency of electron to neutral gas molecules, and $n$ is the neutral gas molecule concentration.

The energy loss of the collision between the electrons and gas molecules per time is $\Delta E$, which is completely converted into the molecule dynamic energy. The energy of the electron can be expressed as follows:

$$
\Delta E=E e \lambda_{e}=\frac{E e k T}{\sqrt{2} \pi d^{2} p}
$$

where $E$ is the applied electrical field, $\lambda_{e}$ is the main free path of electron, and $d$ is the effective diameter of the air molecule.

Given that most air molecules are binary molecules, the average molecule dynamic energy of air molecule is proportional to the thermodynamic temperature:

$$
\Delta E=\frac{7}{2} k \Delta T
$$

Given that arc surface temperature is significantly higher than that of the surroundings, the temperature difference between the arc and the surroundings is approximate to arc surface temperature, viz. $\Delta T \approx T_{S}$. The energy loss of electron per unit and the gas molecule temperature meets the relationship: 


$$
n_{e} \gamma_{e m} \Delta E=n \frac{7}{2} k T_{s}
$$

Considering Eqs. (23) to (25), we can express the gas molecule thermodynamic temperature on the arc surface caused by electron collision as follows:

$$
T_{s}=\frac{\sqrt{2} n_{e} \gamma_{e m} E e T}{7 \pi d^{2} n k}
$$

The ratio of gas molecule thermodynamic temperature at low air pressure and the atmospheric pressure caused by electron collision can be expressed as follows:

$$
\frac{T_{s}}{T_{s 0}}=\frac{n_{e} \gamma_{e m} n_{0} T p_{0}}{n_{e 0} \gamma_{e m 0} n T_{0} p}
$$

where $T_{s 0}, n_{e 0}, \gamma_{e m 0}$, and $n$ are the gas molecule thermodynamic temperature on the arc surface caused by electron collision, the electron density in the arc, the collision frequency of electron with neutral gas molecule, and the neutral gas molecule density, respectively, at temperature $T_{0}$ and air pressure $p_{0}$. Moreover, $T_{s}, n_{e}, \gamma_{e m}$, and $n$ are those at temperature $T$ and air pressure $p$.

Substituting Eqs. (21) and (22) into Eq. (27), we obtain Eq. (28):

$$
\frac{T_{s}}{T_{s 0}}=\left(\frac{p}{p_{0}}\right)^{-0.07} \cdot \frac{T}{T_{0}}
$$

The relationship between the gas molecule thermodynamic temperature on the arc surface caused by electron collision and altitude is shown in Fig. 5, where the gas molecule thermodynamic temperature on the arc surface exhibits a linear decrease with increments of altitude. However, the temperature of the arc surface did not change significantly. When the altitude was $3000 \mathrm{~m}$, the arc surface temperature only dropped by approximately $3.6 \%$.

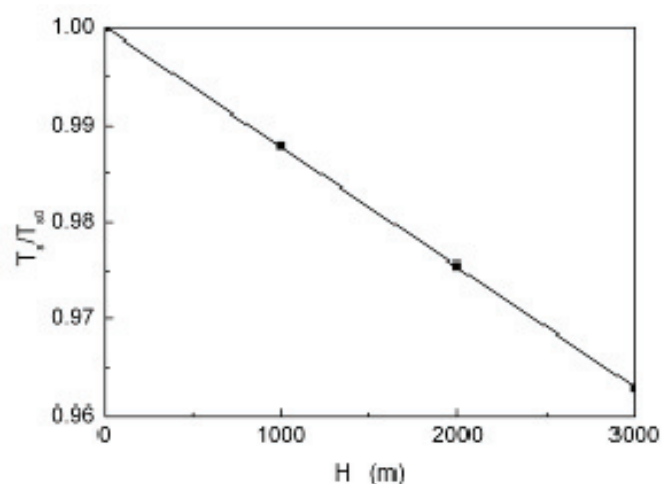

Fig. 5. Relationship between altitude and arc surface temperature caused by electron collision

\subsection{Effect of altitude on the radiation of partial arc}

Although the thermodynamic temperature generated by the electron collision on the arc surface dropped about $3.6 \%$, the arc radiation dropped significantly because arc radiation constant is proportional to the biquadratic order of arc temperature. Supposing that the radiation function and grey body factor of arc are constant, the arc thermal radiation satisfies the following equations:

$$
\begin{gathered}
\frac{U}{U_{0}}=\left(\frac{p}{p_{0}}\right)^{m} \\
\frac{Q_{2}}{Q_{20}}=\frac{C}{C_{0}} \\
\frac{Q_{2}}{Q_{20}}=\left(\frac{p}{p_{0}}\right)^{-0.28}\left(\frac{T}{T_{0}}\right)^{4}
\end{gathered}
$$

According to Eq. (31), the variation of the arc radiation energy with different altitudes is shown in Fig. 6. The thermal radiation capacity of the arc exhibited a linear decrease trend with increments of altitude, which is similar to the trend of thermal conduction capacity. In addition, the thermal radiation of arc dropped for approximately $85 \%$ when the altitude was $3000 \mathrm{~m}$.

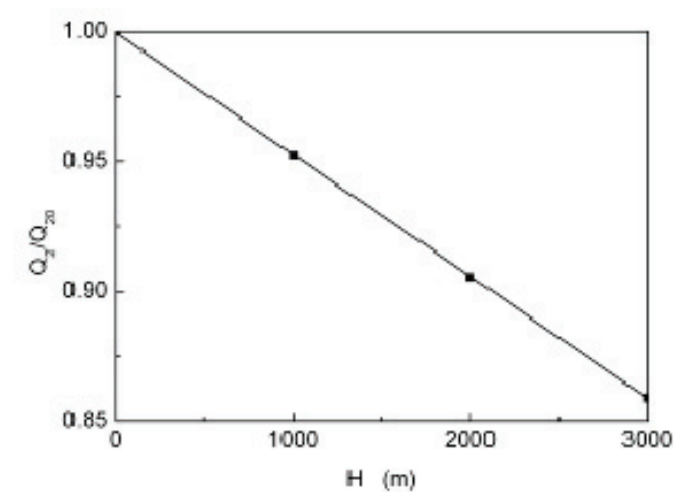

Fig. 6. Relationship between arc thermal radiation value and altitude

\section{Modification of DC Flashover Voltage at High Altitude and Models Comparison}

Both the thermal conduction and radiation of the arc exhibited a linear decrease as shown in Figs. 4 and 6, which indicated that a smaller applied power can maintain the arc movement. Hence, the flashover voltage can be reduced at low pressure.

The most important engineering concern is the flashover voltage. Thus, the effect of low pressure on the flashover voltage is investigated in this study. The above research stated that both the thermal conduction and the thermal radiation of the arc exhibited the same linear downtrend with increments of altitude, and the external energy 


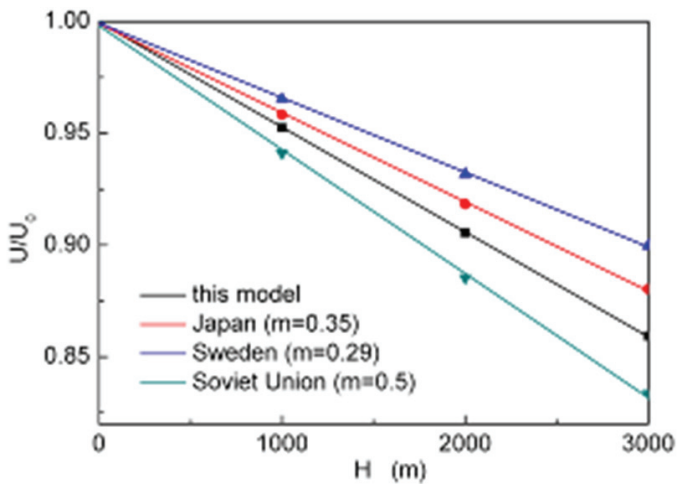

Fig. 7. Relationship between critical flashover voltages and altitudes with different $m$ values

exchange of arc can be substituted by the variation of thermal radiation. Based on the model proposed in this paper, the critical flashover voltage at low pressure meets the following equation:

$$
\frac{U}{U_{0}}=\left(\frac{p}{p_{0}}\right)^{-0.28} \cdot\left(\frac{T}{T_{0}}\right)^{4}
$$

Early studies [1, 13] show that the DC pollution flashover voltage at low pressure satisfies the following relationship:

$$
\frac{U}{U_{0}}=\left(\frac{p}{p_{0}}\right)^{m}
$$

where $U$ and $U_{0}$ are the DC flashover voltages with altitudes $H$ and zero, respectively; $p$ and $p_{0}$ are the air pressure values with altitudes of $H$ and zero, respectively; and $m$ is the air pressure (altitude) influence characteristic exponent.

According to Eqs. (32) and (33), the change relation of negative polarity critical flashover voltage and the altitude with different $\mathrm{m}$ value is shown in Fig. 7. The critical flashover voltages obtained from the different models all exhibited linear decrements with increments of altitude. Moreover, the model proposed in this study reflects that the critical flashover voltage is not only associated with pressure, but also with the temperature in the corresponding altitude, which is significant for the deep understanding of the pollution flashover mechanism at low pressures.

\section{High Altitude Simulation Experiment}

\subsection{Specimens}

The experiment was conducted with the use of the common toughened glass flat model, and three glass flats with the size of $20 \mathrm{~cm} \times 20 \mathrm{~cm} \times 1 \mathrm{~cm}$. A column-plate electrode was used to be consistent with the electric field

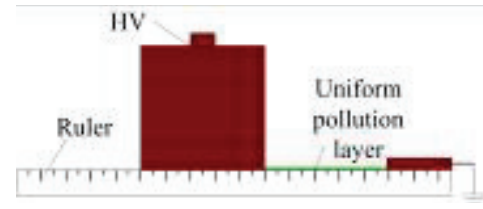

(a)

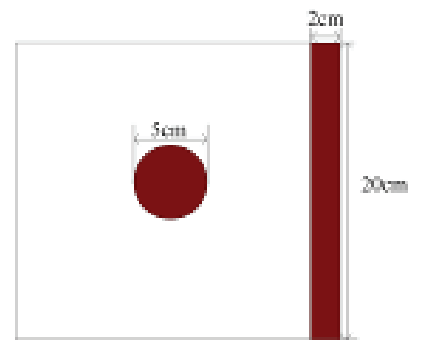

(b)

Fig. 8. Schematic diagram of the specimen

structure of the Obenaus model. On the left is a cylindrical copper high voltage electrode with a height and diameter 5 $\mathrm{cm}$, and the distance of the high voltage electrode surface to the left and right ends are both $7.5 \mathrm{~cm}$. On the right is the rectangular grounding electrode with length, width, and height of 2,20 , and $0.2 \mathrm{~cm}$, respectively. A ruler was stuck to the side of the glass plate to observe the distance of the arc development anytime. The upright and bottom views of the sample are shown in Figs. 8 (a) and 8 (b).

\subsection{Experiment equipment}

\subsubsection{Main experiment equipment}

The main performance parameters of the regulator were as follows: rated capacity of $100 \mathrm{kVA}$, input voltage of 380 $\mathrm{V}$, and output voltage of $400 \mathrm{~V}$. The main performance parameters of the transformer were as follows: rated capacity of $200 \mathrm{kVA}$, rated current of $1 \mathrm{~A}$, and input and output voltages of $0 \mathrm{~V}$ to $400 \mathrm{~V}$ and $0 \mathrm{kV}$ to $200 \mathrm{kV}$, respectively. The DC power was supplied by silicon stack rectification. The dynamic voltage fall was lower than $5 \%$ when the leakage current was $1 \mathrm{~A}$, and the voltage ripple coefficient was lower than 3\% during the flashover, both of which all meet the requirement of DC pollution testing power of IEC 61245 [23]. The main experiment equipment is shown in Fig. 9.

The experiment power source was introduced to the laboratory through a wall bushing, and the applied voltage and leakage current were collected synchronously using a NI USB-6525 data acquisition card. In the acquisition process, the applied voltage was measured using a resistance voltage divider with the attenuation ratio of $1: 10000$, and the leakage current was realized by measuring the $1 \Omega$ noninductance resistor, which is in series between the grounding electrode and the earth. Protection resistances R1 and R2 were applied to prevent the current from 


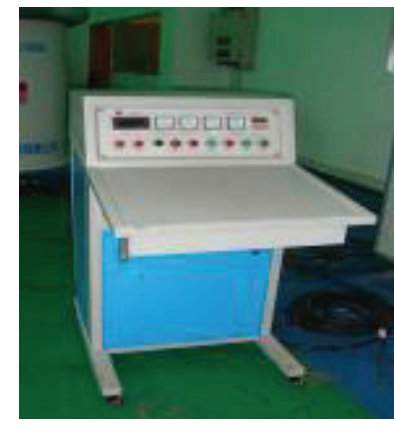

(a) Regulator control cabinet

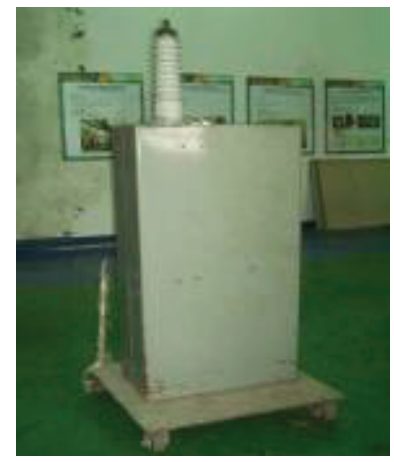

(c) Capacitor

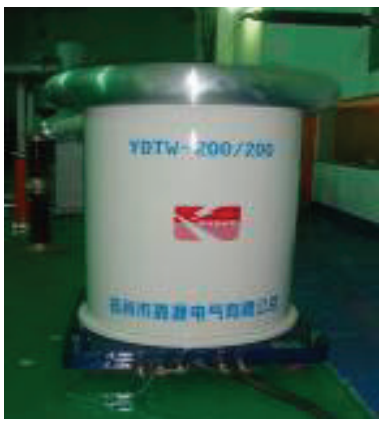

(b) Regulator

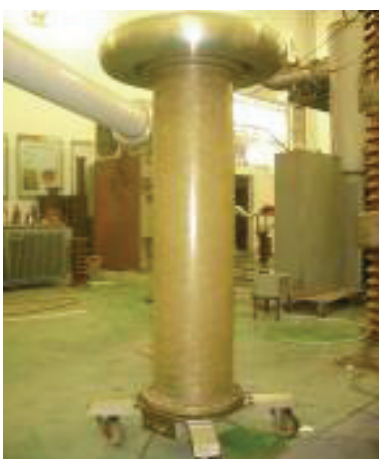

(d) Standard DC divider

Fig. 9. Main experiment equipment

becoming too large in the experiment. $\mathrm{R} 1$ is $5 \mathrm{k} \Omega$, and $\mathrm{R} 2$ is water resistance with conductivity lower than $5 \mu \mathrm{S} \cdot \mathrm{cm}^{-1}$.

\subsubsection{Equipment of high altitude simulation and experi- ment wiring diagram}

The high altitude condition was simulated by controlling the pressure of a sealed container to validate the proposed calculation model of critical flashover voltage at high altitude. The sealed container and steam generator used in the experiment are shown in Fig. 10. The sealed container is made of stainless steel with a height and diameter of 765 and $800 \mathrm{~mm}$, respectively. The humidity can be controlled from $30 \%$ to $100 \%$, and the pressure can be controlled from $30 \mathrm{kPa}$ to $101.3 \mathrm{kPa}$. The power of the sealed

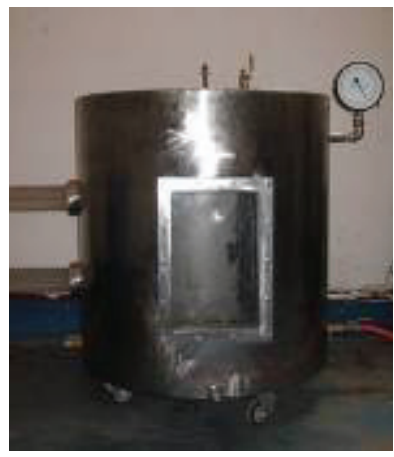

(a) Closed container

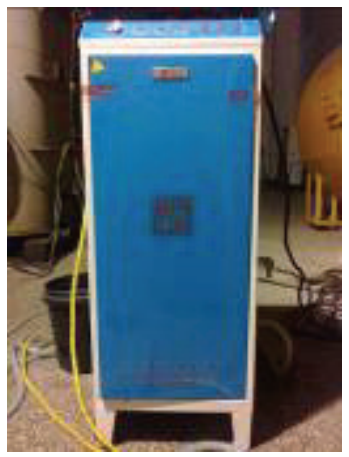

(b) Steam generator

Fig. 10. The closed container and steam generator

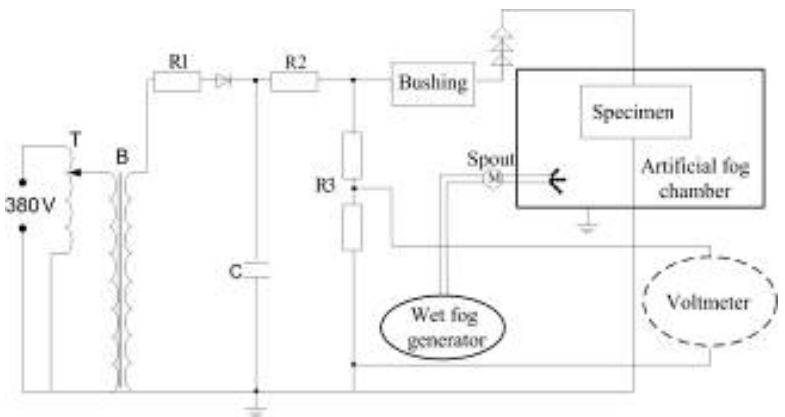

T-Regulator; B-Transformer; R1/R2-Protection resistances; C-Filter capacitor; R3-Standard DC divider

Fig. 11. Schematic diagram of high altitude experiment circuit

container is $9 \mathrm{~kW}$, and the total spray water volume can be controlled from 0 to $12.9 \mathrm{~kg} / \mathrm{h}$. The specimen, sealed container, and experiment wiring diagram are shown in Fig. 11.

\subsubsection{Experiment results and analysis}

In order to study the statistic condition and the variation of critical flashover voltage, 10 times of flashover experiment have been carried out with different pollution degree and air pressure respectively. The interval of two test is 5 minutes. Take the value of error below $10 \%$ with average value as an effective test value, and the final critical flashover voltage is the average value of all the effective test values.

The variations between pollution flashover voltage and altitude with the different pollution degrees were calculated according to the practical condition of engineering, and a simulation experiment was conducted. Figs. 12-14, and 15 are the critical flashover voltage comparisons of calculations and experiments with altitudes of $0,1000,2000$, and 3000 $\mathrm{m}$, respectively. The critical flashover voltage exhibited a parabola decrease trend with increments of pollution degree, and the calculation value of critical flashover voltage was consistent with that of the experiment, especially when

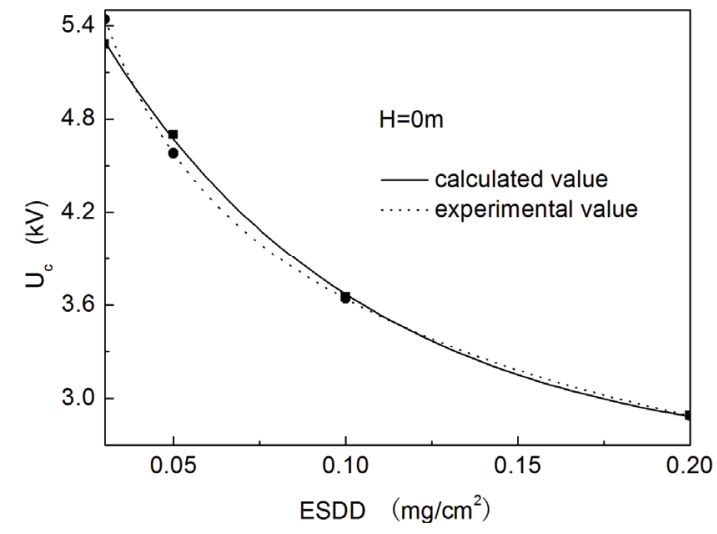

Fig. 12. Calculated value and experimental value of critical flashover voltage at $0 \mathrm{~m}$ 


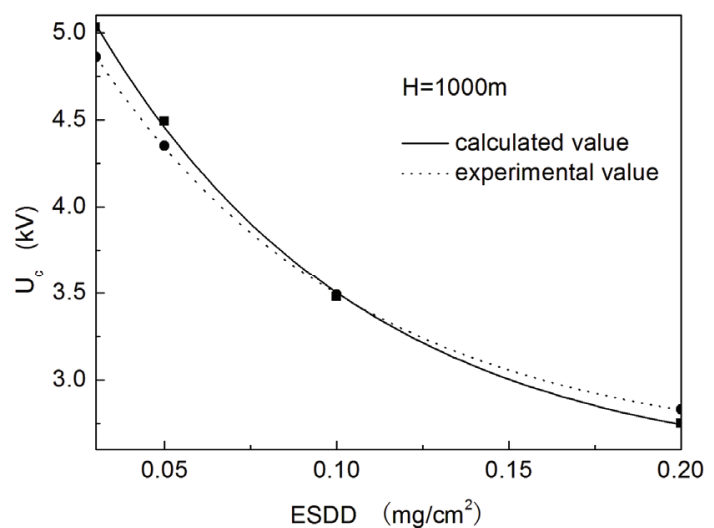

Fig. 13. Calculated value and experimental value of critical flashover voltage at $1000 \mathrm{~m}$

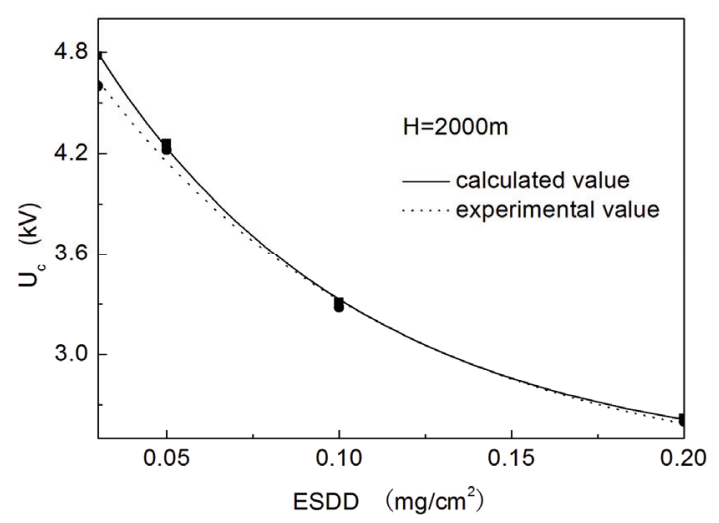

Fig. 14. Calculated value and experimental value of critical flashover voltage at $2000 \mathrm{~m}$

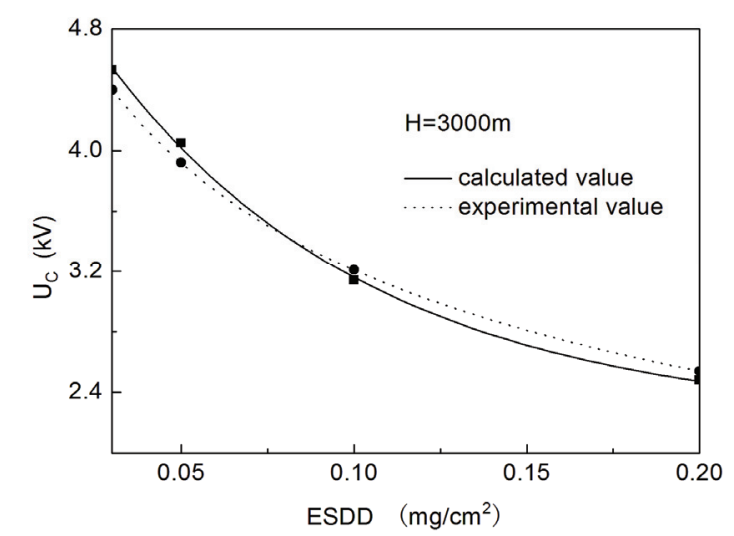

Fig. 15. Calculated value and experimental value of critical flashover voltage at $3000 \mathrm{~m}$

the altitude was at 0 and $3000 \mathrm{~m}$, and the maximum error was only approximately $2.9 \%$. And the maximum error at 1000 and $2000 \mathrm{~m}$, was less than $6.6 \%$.

\section{Conclusion}

The effect of altitude on the arc thermal conduction, arc surface temperature, arc thermal radiation, and DC flashover voltage was investigated on the basis of the arc energy balance flat model with consideration of the radiation and the molecular gas dynamics. The main conclusions are as follows:

(1) The arc thermal conduction coefficient exhibited a linear decrease trend with increments of altitude. The thermal conduction coefficient dropped by approximately $30 \%$ when the altitude increased from $0 \mathrm{~m}$ to $3000 \mathrm{~m}$, whereas the arc thermal conduction dropped only by approximately $15 \%$.

(2) Both the arc surface thermodynamics temperature and the arc thermal radiation capability exhibited linear decreases with increments of altitude. The arc surface thermodynamics temperature dropped by approximately $3.6 \%$ when the altitude increased from $0 \mathrm{~m}$ to $3000 \mathrm{~m}$, whereas the arc thermal radiation dropped by approximately $15 \%$, which is the same as the case of arc thermal conduction.

(3) The DC flashover voltage is a function of pressure and temperature at high altitude and satisfied the relationship: $U / U_{0}=\left(p / p_{0}\right)^{-0.28 .}\left(T / T_{0}\right)^{4}$.

(4) With increments of altitude, the maximum error of DC flashover voltage between the calculation value obtained from the proposed model and the experiment value was less than $6.6 \%$.

\section{Acknowledgements}

This work was supported by the Funds for Innovative Research Groups of China (51021005) and the National Basic Research Program of China (973 Program) (2009CB 724504).

\section{References}

[1] Caixin Sun. Atmospheric environment and Electrical insulation. Beijing: China power press, 2002, pp. 3546.

[2] M. EI-A. Slama, A. Beroual, H. Hadi. Analytical computation of discharge characteristic constants and critical parameters of flashover of polluted insulators. IEEE Transactions on Electrical Insulation, 17, pp. 1764-1771, 2010.

[3] Rizk F A M. Mathematical models for pollution flashover. Electra, 78, pp. 71-103, 1981.

[4] Sundararajan R, Gorur R S. Dynamic arc modeling of pollution flashover if insulators under DC voltage. IEEE Trans. on Electrical Insulation, vol. 26, no. 2, pp. 209-218, 1993.

[5] Fusheng Guo, Wenxia Sima, Qing Yang, Tao Yuan. Calculation of the physical characteristics of development streamer at arc head along the polluted surface. International Review of Electrical Engi- 
neering, vol. 7, no. 2, pp. 4343-4350, 2012.

[6] Wenxia Sima, Fusheng Guo, Qing Yang, Tao Yuan, Rui Wang. Radiant discharge development model for polluted surface along short plate under DC voltage. Proceedings of the CSEE, vol. 32, no. 22, pp. 174181, 2012.

[7] Wenxia Sima, Fusheng Guo, Qing Yang, Tao Yuan. Calculation of the arc velocity along the polluted surface of short glass plates considering the air effect. Energies, vol.5, no.3, pp. 815-834, 2012.

[8] Zhang Zhijin, Jiang Xingliang, SunCaixin, Hu Jianlin, Yuan Jihe. DC Pollution flashover process for insulator string at low air pressure. Transactions of China Electro-technical Society, vol. 24, no. 4, pp. 30-35, 2009.

[9] Rudakova v m, Tikhodeev N N. Influence of low air pressure on flashover voltage of polluted insulators: test data, generalization attempts and some recommendations. IEEE Transactions on Power Delivery, vol.4, no.1, pp. 607-613, 1989.

[10] Bergman V I, Kolobova O I. Some results of investigation of the dielectric strength of polluted lines insulation in conditions of reduced atmospheric. Electrotrchnika, vol. 54, no. 2, pp. 54-56, 1983.

[11] Kawamura T, Ishii M, Akbar M, et al. Pressure dependence of DC breakdown of contaminated insulators. IEEE Transactions on Electrical Insulation, EI-vol.17, no.1, pp. 39-45, 1982.

[12] Xingliang Jiang, Jihe Yuan, Lichun Shu etc. comparison of DC pollution flashover performances of various types of porcelain, glass and composite insulators. Power Delivery, vol. 23, no. 2, pp. 1183-1190, 2008.

[13] Zhicheng Guan. External insulation of insulator and Power Transmission Equipment. Beijing: Tsinghua University press, 2006, pp. 115-132.

[14] Xu Jialuan, Jin Shangxian. Plasma physics. Atomic Energy Press: Beijing, China, 1981, pp. 169-176.

[15] Li Shunyuan, Zhang Renyu, Tan Kexiong. The study of electric arc propagating along a polluted dielectric surface under AC voltage. Proceedings of the CSEE, vol. 11, no. 2, pp. 1-7, 1991.

[16] Guo Zengyuan, Zhao Wenhua. Arc and thermal plasma. Science Press: Beijing, China, 1986, pp. 10-60.

[17] Dai, G. Heat Transfer, 2nd ed.; Higher Education Press: Beijing, China, 1999, pp. 188-206.

[18] Zhang Zhijin, Jiang Xingliang, Sun Caixin, Hu Jianlin, Yuan Jihe. DC pollution flashover model and its validation of polluted insulator strings. Transactions of China electrotechnical society, vol. 24, no. 4, pp. 36-41, 2009.

[19] Zhang Lanzhi. Thermology. Harbin Institute of Technology Press: Harbin, China, 2000, pp. 223-224.

[20] Guan Zhicheng, Zhang Renyu. Calculation of dc and ac flashover voltage of polluted insulators. IEEE Transactions on Electrical Insulation, vol. 25, no. 4, pp. 723-729, 1990.
[21] Williams D L, Haddad A, Rowlands A R, et al. Formation and characterization of dry bands in clean fog on polluted insulators. IEEE Transactions on Dielectrics and Electrical Insulation, vol. 6, no. 5, pp. 724-731, 1999.

[22] Y. Itikawa. Effect collision frequency of electrons in gases. Phys. Fluids, 16, pp. 831-835, 1973.

[23] Artificial pollution tests on high-voltage insulators to be used on d. c. systems, IEC Tech. Rep. 61245, 1993.

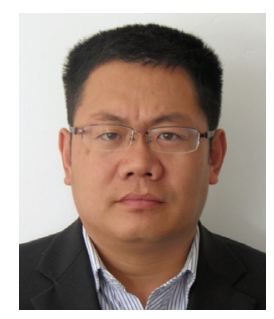

Dong-Ming Liu He received his M.S. degree in electrical engineering from Shandong University in 1998. His research interests include relay protection, high-voltage and insulation technology.

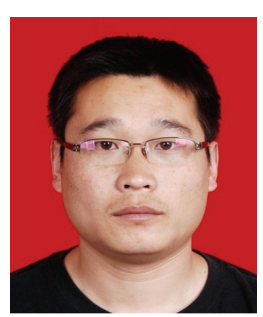

Fu-sheng Guo He received his Ph.D. degree in electrical engineering from Chongqing University in 2012. His research interests include high-voltage external insulation discharge characteristics and mechanism in special environment.

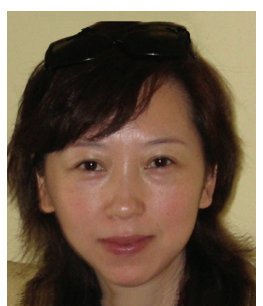

Wen-xia Sima She received her Ph.D. degree in electrical engineering from Chongqing University in 1994. Her research interests include high-voltage external insulation discharge characteristics and mechanism in special environment, and power system lighting and over-voltage protection. 